\title{
Methotrexate improves perivascular adipose tissue/endothelial dysfunction via activation of AMPK/eNOS pathway
}

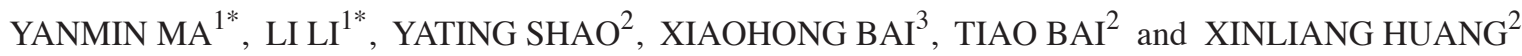 \\ Departments of ${ }^{1}$ Pharmacy and ${ }^{2}$ Gerontology, Ninth Hospital of Xi'an, Xi'an, Shaanxi 710054; \\ ${ }^{3}$ Department of Pharmacy, Chang'an District Hospital of Xi'an, Xi'an, Shaanxi 710018, P.R. China
}

Received November 25, 2015; Accepted November 29, 2016

DOI: $10.3892 / \mathrm{mmr} .2017 .6225$

\begin{abstract}
Adipose and endothelial dysfunction is associated with cardiovascular disease. Perivascular adipose tissue (PVAT) directly surrounds vessels and influences vessel function via a paracrine effect, and adenosine monophosphate (AMP)-activated protein kinase (AMPK) modulates the metabolic pathway, thus, the present study hypothesized that activation of AMPK in PVAT may regulate endothelial function in pathological settings. The present study investigated the effect of methotrexate (MTX) on adipocytokine expression in PVAT with an emphasis on the regulation of endothelial function. The effects of MTX and the mechanisms involved were investigated using a relaxation assay and western blot analysis. Reverse transcription-quantitative polymerase chain reaction and western blotting were used to detect the mRNA and protein expression levels. ELISA assay was used to quantify the level of TNF- $\alpha$ and IL-6. Palmitic acid (PA) stimulation induced inflammation and dysregulation of adipocytokine expression in PVAT. MTX treatment inhibited nuclear factor- $\kappa \mathrm{B}$ p65 phosphorylation and downregulated expression of pro-inflammatory cytokines, including tumor necrosis factor- $\alpha$ and interleukin- 6 , whereas adiponectin expression increased. MTX increased AMPK phosphorylation under basal and inflammatory conditions in PVAT, whereas knockdown of AMPK via small interfering RNA diminished its modulatory effect, indicating that MTX inhibits inflammation in an AMPK-dependent manner. The present study prepared conditioned medium from PA-stimulated PVAT to induce endothelial dysfunction and observed that pre-treatment of PVAT with MTX effectively restored the
\end{abstract}

Correspondence to: Dr Yating Shao, Department of Gerontology, Ninth Hospital of Xi'an, 151 Youyi Dong Road, Xi'an, Shaanxi 710054, P.R. China

E-mail: yatingshao@tom.com

*Contributed equally

Key words: methotrexate, adenosine monophosphate-activated protein kinase/endothelial nitric oxide synthase, perivascular adipose tissue, endothelial dysfunction loss of acetylcholine-induced vasodilation and increased endothelial nitric oxide synthase phosphorylation in the rat aorta. The results of the present study demonstrated that MTX ameliorated inflammation-associated adipocytokine dysregulation and thus prevented endothelial dysfunction. These data provide further pharmacological evidence regarding the beneficial effects of MTX in cardiovascular diseases.

\section{Introduction}

Inflammation results in the initiation and progression of atherosclerosis. Atherosclerosis is the primary cause of cardiovascular disease and conditions that affect the cerebral, coronary and peripheral vasculature, and is the most common cause of morbidity and mortality worldwide (1). Endothelial dysfunction is characterized by reduced endothelial nitric oxide (NO) synthase (eNOS)-derived NO bioactivity, and the impairment of endothelium-dependent vascular dysfunction is the progenitor of atherogenesis (2). Approaches designed to improve endothelial function are expected to have therapeutic value in the prevention or treatment of atherosclerosis (3). Perivascular adipose tissue (PVAT) directly surrounds vessels and influences their function via a paracrine effect.

Adenosine monophosphate-activated protein kinase (AMPK) is an important regulator of energy metabolic homeostasis and emerging evidence demonstrates its anti-inflammatory action in vessel and adipose tissue $(4,5)$. AMPK may modulate a number of signaling cascades that are expected to have anti-endothelial cell dysfunction, including the attenuation of free radicals (6). In a preliminary experiment, the authors demonstrated that pharmacological activation of AMPK beneficially regulated adipocytokine expression in PVAT against inflammatory insult and ameliorated endothelial dysfunction. These findings demonstrated the role of AMPK activation in the regulation of PVAT and endothelial function (7).

Methotrexate (MTX), a non-specific anti-inflammatory therapy, may be an ideal agent to directly test the inflammatory hypothesis of atherosclerosis, as it inhibits inflammation with only minimal impact on other components of the atherosclerotic process and exhibits an acceptable safety profile $(8,9)$. A recent pre-clinical study with cholesterol-fed rabbits revealed that MTX markedly reduced atherosclerosis, without effects on plasma lipid and lipoprotein levels (10). The Cardiovascular 
Inflammation Reduction Trial evaluates the use of very low-dose MTX on cardiovascular events and plasma lipid levels in coronary artery disease patients with elevated C-reactive protein levels and is expected to offer more conclusive results in the future (9).

The present study investigated the effect of MTX on AMPK activation and adipocytokine expression in PVAT with emphasis on the regulation of endothelial function. It was observed that MTX ameliorated endothelial dysfunction by inhibiting inflammation in PVAT. These findings provide novel information regarding the underlying mechanism of MTX in the management of cardiovascular diseases.

\section{Materials and methods}

Animals. Sprague-Dawley male rats $(\mathrm{n}=24 ; 8$-weeks old; weight, 200-250 g) were supplied by the Laboratory Animal Center of Nanjing Qinglongshan (Nanjing, China). The care and treatment of these rats was performed in accordance with the Provisions and General Recommendation of Chinese Experimental Animals Administration Legislation. All animals were housed in a room with a constant temperature $\left(22 \pm 1^{\circ} \mathrm{C}\right)$ and a humidity of $50 \pm \%$, allowed access to a standard diet and water ad libitum, and exposed to a 12-h light/dark cycle. The present study was approved by the Ethics Committee of Ninth Hospital of Xi'an (Xi'an, China).

Preparation of PVAT-derived conditioned medium (CM). Sprague-Dawley rats were anesthetized with diethyl ether and sacrificed by cervical dislocation. PVAT (located around the thoracic aorta) was isolated, sectioned into small pieces and rinsed in phosphate buffered saline (PBS). Equal quantities $(40 \mathrm{mg})$ of PVAT were individually pretreated with MTX (Sigma-Aldrich; Merck Millipore, Darmstadt, Germany) $(0.1,0.25$ and $0.5 \mu \mathrm{M})$ or salicylate $(5 \mathrm{mM}$; Tianjin Kemiou Chemical Reagent Co., Ltd., Tianjin, China) or aminoimidazole-4-carboxamide ribonucleotide (AICAR; $500 \mu \mathrm{M}$; Sigma-Aldrich, Merck Millipore) in the presence or absence of $25 \mu \mathrm{M}$ AMPK inhibitor compound C (Sigma-Aldrich; Merck Millipore) and stimulated with $100 \mu \mathrm{M}$ palmitic acid (PA; Sinopharm Chemical Reagent Co., Ltd., Shanghai, China) for $2 \mathrm{~h}$. Following treatment, the PVAT was washed twice with PBS to remove the reagents, and cultured in fresh Dulbecco's modified Eagle's medium (DMEM, Gibco; Thermo Fisher Scientific, Inc., Waltham, MA, USA) supplemented with $10 \%$ $(\mathrm{v} / \mathrm{v})$ fetal bovine serum (FBS) for $22 \mathrm{~h}$ at $37^{\circ} \mathrm{C}$. The medium was collected as $\mathrm{CM}$. The supernatant was then collected as conditioned $\mathrm{CM}$ and stored at $-70^{\circ} \mathrm{C}$.

Endothelium-dependent relaxation assessment. Endothelium-dependent relaxation was assayed as previously described (7). Briefly, the prepared aortic ring was suspended in an organ bath containing $10 \mathrm{ml} \mathrm{K}-\mathrm{H}$ solution $(118.3 \mathrm{mM}$ $\mathrm{NaCl}, 4.7 \mathrm{mM} \mathrm{KCl}, 1.2 \mathrm{mM} \mathrm{MgSO}_{4}, 1.2 \mathrm{mM} \mathrm{KH}_{2} \mathrm{PO}_{4}$, $2.5 \mathrm{mM} \mathrm{CaCl}_{2}, 25 \mathrm{mM} \mathrm{NaHCO}{ }_{3}, 0.026 \mathrm{mM}$ calcium disodium EDTA and $5.0 \mathrm{mM}$ glucose, $\mathrm{pH}$ 7.4) maintained at

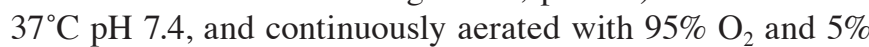
$\mathrm{CO}_{2}$. Following a $60 \mathrm{~min}$ stabilization period, the contractive ability of the vessel was examined by contractive response to $60 \mathrm{mM} \mathrm{KCl}$, and the functionality of vascular endothelium was confirmed by relaxation to $10 \mu \mathrm{M}$ acetylcholine (ACh; Sigma-Aldrich; Merck Millipore). The aortic ring (relaxation, $\geq 80 \%$ ) was treated with CM for $30 \mathrm{~min}$. Following washing, the aortic ring was pre-contracted with phenylephrine and the endothelium-dependent relaxation was induced by cumulative addition of ACh (0.001-10 $\mu \mathrm{M})$. Relaxation was expressed as a percentage of the phenylephrine-induced contraction.

Cell culture. 3T3-L1 pre-adipocytes were purchased from the Cell Bank of the Chinese Academy of Sciences (Shanghai, China). A total of $2 \times 10^{5} / \mathrm{ml}$ cells were cultured in 6-well plates in DMEM containing $10 \% \mathrm{FBS}$ and $25 \mathrm{mM}$ glucose at $37^{\circ} \mathrm{C}$ in a humidified atmosphere containing $5 \% \mathrm{CO}_{2}$.

RNA extraction and reverse transcription-quantitative polymerase chain reaction (RT-qPCR). PVAT from rats were pretreated with MTX in the presence of PA $(100 \mu \mathrm{M})$ for $2 \mathrm{~h}$. Following washing with PBS, PVAT was cultured in fresh DMEM supplemented with $10 \%$ (v/v) FBS for $22 \mathrm{~h}$. PVAT was then collected and homogenized with $500 \mu \mathrm{IRIzol}^{\circledR}$ (Invitrogen; Thermo Fisher Scientific, Inc.) on ice. Total RNA was obtained using Total RNA Extraction Reagent (Nanjing Sunshine Biotechnology Co., Ltd., Nanjing, China). cDNA was synthesized with the TransScript First-strand cDNA Synthesis SuperMix kit (Beijing Transgen Biotech Co., Ltd., Beijing, China). qPCR was performed using SsoFast ${ }^{\mathrm{TM}}$ EvaGreen Supermix (Bio-Rad Laboratories Inc., Hercules, CA, USA). Amplification was performed with the Bio-Rad iQ5 sequence detection system (Bio-Rad Laboratories Inc.) with the following conditions: $95^{\circ} \mathrm{C}$ for $10 \mathrm{~min}$, followed by 40 cycles at $95^{\circ} \mathrm{C}$ for $30 \mathrm{sec}$, and $52^{\circ} \mathrm{C}$ for $15 \mathrm{sec}$, and a final extension at $72^{\circ} \mathrm{C}$ for $5 \mathrm{~min}$. The following primers were used: Adiponectin forward, 5'-AAGGGGACAACAATGGAC TCTA-3', and reverse, 5'-CTACGGGCTGCTCTGAATTAG T-3'; $\beta$-actin forward, 5'-GACGTTGACATCCGTAAAGAC C-3', and reverse, 5'-TGCTAGGAGCCAGGGCAGTA-3'. The mRNA expression level of individual genes was normalized and presented as a ratio to $\beta$-actin, and calculated using the $2^{-\Delta \Delta C q}$ method (11).

Western blot analysis. PVAT from Sprague-Dawley rats was pretreated with MTX for 30 min and then incubated with PA for a further $30 \mathrm{~min}$. The aorta was incubated with $\mathrm{CM}$ for $30 \mathrm{~min}$ and then exposed to ACh for another 30 min. PVAT was isolated directly following the sacrifice of the rats. For protein analysis, PVAT or the aorta was homogenized in radio immunoprecipitation assay lysis buffer with phenylmethane sulfonyl fluoride. Protein concentration in the supernatants was assayed using Bicinchoninic Acid Protein Assay kit (Nanjing Baxi Technology Co., Ltd. Nanjing, China). Equal amounts of protein $(30 \mu \mathrm{g})$ were separated by $10 \%$ SDS-PAGE and transferred to $0.45 \mu \mathrm{M}$ polyvinylidene difluoride (PVDF) membranes (EMD Millipore, Billerica, MA, USA) by semi-dry electrophoretic transfer (Bio-Rad Laboratories, Inc.). The PVDF membranes were blocked with 5\% non-fat milk in Tris-buffered saline with Tween-20 (TBST) and then incubated with the appropriate primary antibodies overnight at $4^{\circ} \mathrm{C}$ : Rabbit monoclonal anti-phospho-AMPK (cat. no. 2531) and anti-AMPK (cat. no. 2532), rabbit monoclonal anti-eNOS (cat. no. 9586) and anti-p-eNOS (cat. no. 9571), anti-NF-kB 
A

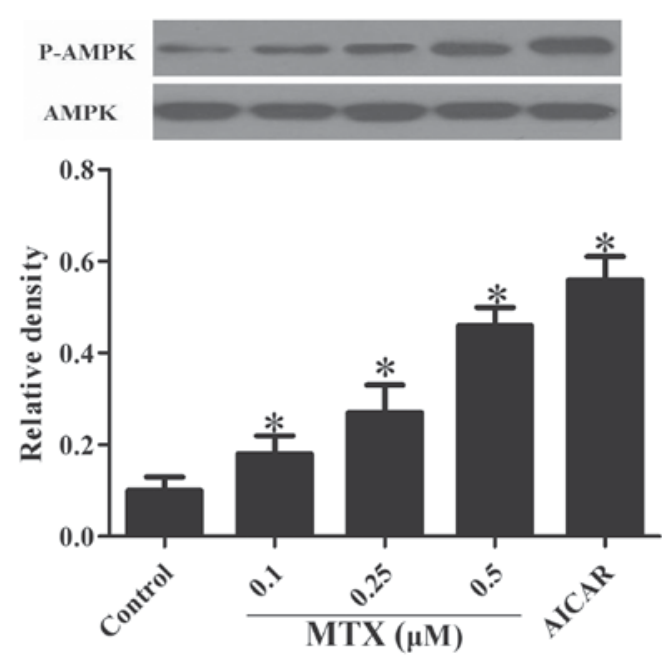

B

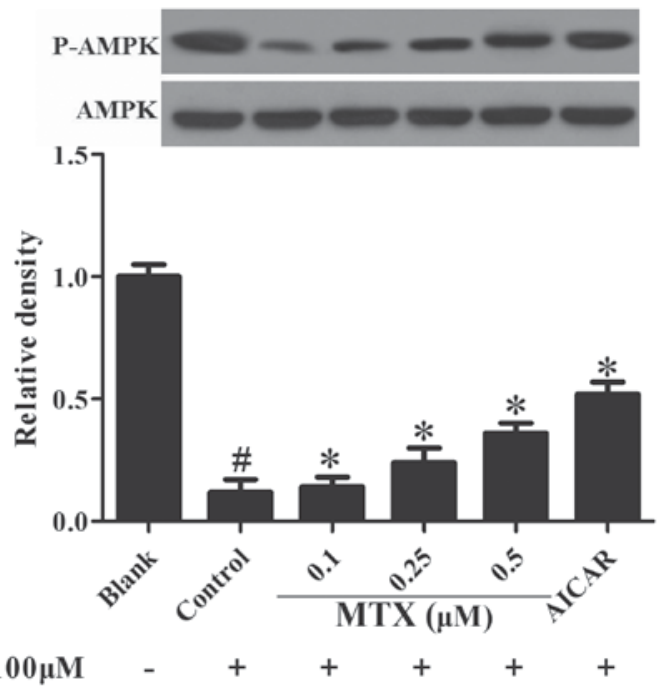

Figure 1. MTX regulated AMPK phosphorylation in PVAT. (A) PVAT incubated with MTX for $1 \mathrm{~h}$. (B) PVAT pretreated with MTX for 30 min, then stimulated with $100 \mu \mathrm{M}$ PA for a further $30 \mathrm{~min}$. AMPK phosphorylation was determined by western blot analysis. AICAR served as a positive control. Data are expressed as the mean \pm standard deviation of three independent experiments. ${ }^{*} \mathrm{P}<0.05$ vs. the control. MTX, methotrexate; PVAT, perivascular adipose tissue; AMPK, adenosine monophosphate-activated kinase; p-AMPK, phosphorylated AMPK; PA, palmitic acid; AICAR, aminoimidazole-4-carboxamide ribonucleotide.

p65 (cat. no. 4764) and anti-p-NF-kB p65 (cat. no. 3033; all at 1:1,000 and obtained from Cell Signaling Technology, Inc., Beverly, MA, USA). The PVDF membrane was washed three times with TBST buffer and then incubated with the HRP-conjugated secondary antibodies (cat. no. BS13278; 1:10,000, MyBioSource, Inc., San Diego, CA, USA) at room temperature for $2 \mathrm{~h}$. The membranes were developed with an enhanced chemiluminescence detection reagent (Thermo Fisher Scientific, Inc.) and quantitated by densitometry with Image-Pro Plus 6.0 software (Media Cybernetics, Inc., Rockville, MD, USA).

ELISA assay. The levels of TNF- $\alpha$ and IL-6 in the supernatant were assayed with commercial enzyme-linked immunosorbent assay (ELISA) kits (R\&D, Minneapolis, MN, USA).

AMPK small interfering (si)RNA transfection. 3T3-L1 pre-adipocytes were transfected with siRNA for AMPK $\alpha$ (cat. no. sc-45313) to knock down levels of endogenous AMPK $\alpha$, and negative control siRNA (cat. no. sc-37007), using siRNA transfection reagent (cat. no. sc-29528) in transfection medium (cat. no. sc-36868) for $6 \mathrm{~h}$, according to the manufacturer's protocol. All siRNAs were obtained from Santa Cruz Biotechnology, Inc., Dallas, TX, USA. Following an additional $48 \mathrm{~h}$, the efficiency of siRNA-mediated AMPK knockdown was confirmed by western blot analysis as aforementioned.

Statistical analysis. Data were expressed as the mean \pm standard deviation from at least three independent experiments. SPSS version 13.0 software (SPSS, Inc., Chicago, IL, USA) was used for the statistical analysis. Individual group statistical comparisons were analyzed using an unpaired Student's $t$-test with Bonferroni correction and multiple-group comparisons were evaluated by one-way analysis of variance followed by Tukey's post-hoc test. $\mathrm{P}<0.05$ was considered to indicate a statistically significant difference.

\section{Results}

$M T X$ regulates AMPK activation in PVAT. AMPK is important in the regulation of lipid metabolism, therefore the present study first investigated the effect of MTX on AMPK activity in PVAT. As presented in Fig. 1A, MTX treatment increased basal AMPK activity at concentrations ranging from 0.1 to $0.5 \mu \mathrm{M}$, indicated by enhanced AMPK phosphorylation. In addition, it was observed that when PVAT was exposed to PA, a reduction of AMPK phosphorylation was observed, and this alteration was prevented by pretreatment with MTX $(\mathrm{P}<0.05$, Fig. 1B). Furthermore, treatment with AICIR which is an AMPK agonist, exhibited similar effects $(\mathrm{P}<0.05)$. These results indicated that MTX enhanced AMPK activation under basal and inflammatory conditions.

MTX inhibits inflammation and modulates adipocytokine expression in PVAT. It has previously been demonstrated that free fatty acids induce inflammation in the endothelium via the nuclear factor (NF) $-\kappa \mathrm{B}$ pathway and stimulation with PA enhanced phosphorylation of NF- $\mathrm{B}$ p65 in PVAT (12). As presented in Fig. 2A, MTX effectively reduced NF- $\kappa$ B p65 phosphorylation, indicative of its anti-inflammatory activity $(\mathrm{P}<0.05)$. In addition, the effect of MTX on the expression of adipocytokines implicated in inflammation was examined. As presented in Fig. 2B and C, the expression levels of pro-inflammatory cytokines, including tumor necrosis factor (TNF)- $\alpha$ and interleukin (IL)-6, were increased in cells treated with PA for $24 \mathrm{~h}$ and this was reversed by MTX pretreatment $(\mathrm{P}<0.05)$, demonstrating its anti-inflammatory activity. Furthermore, the expression of adiponectin was downregulated, however these alterations were reversed by treatment with MTX $(\mathrm{P}<0.05$, Fig. 2D).

AMPK silencing blocks the anti-inflammatory effect of MTX. The AMPK inhibitor to confirm the role of AMPK in the 

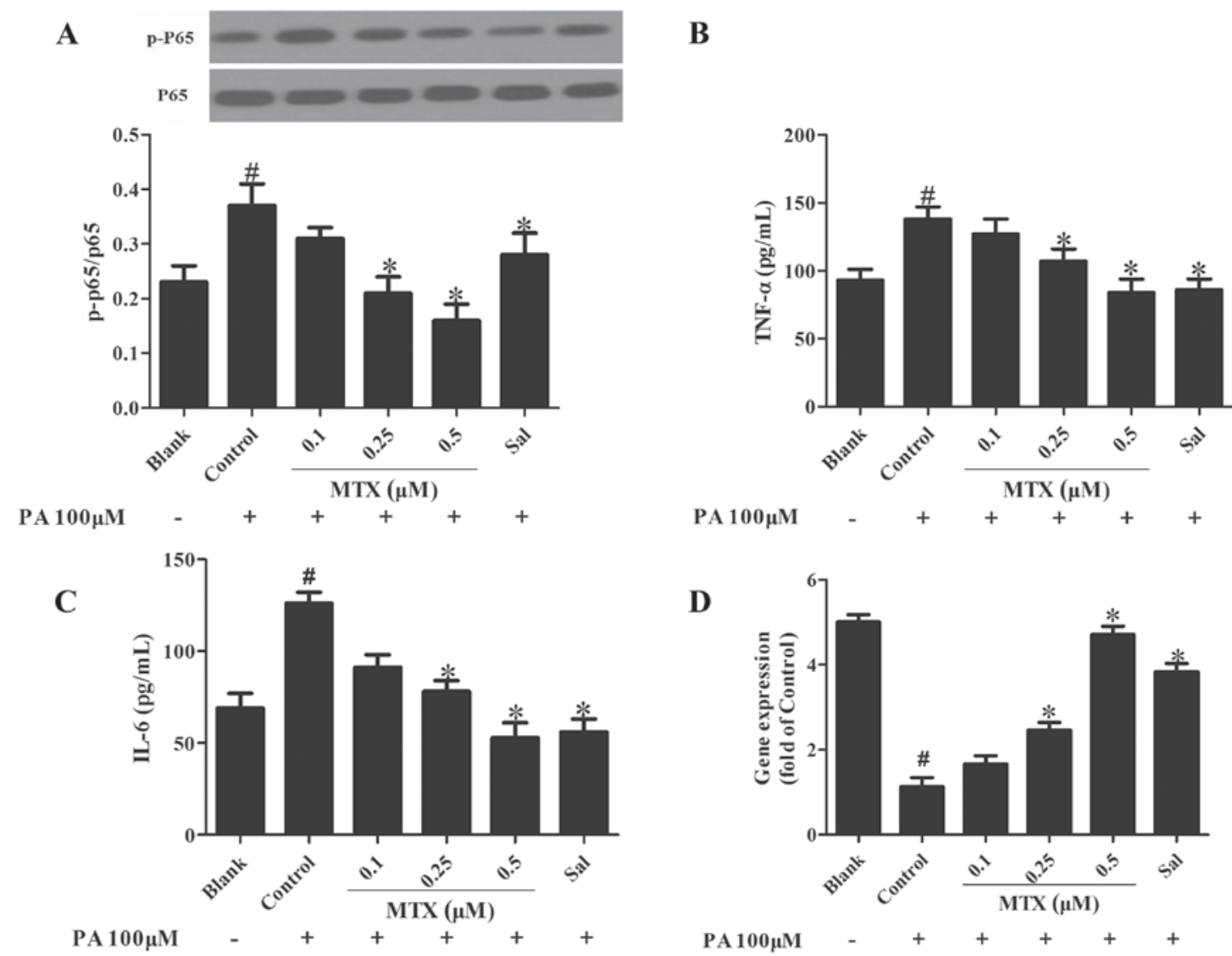

Figure 2. MTX suppresses nuclear factor- $\mathrm{kB}$ activation and regulates adipocytokine expression in perivascular adipose tissue. (A) Cells pretreated with MTX or $5 \mathrm{mM}$ Sal for $30 \mathrm{~min}$, followed by $30 \mathrm{~min}$ treatment with $100 \mu \mathrm{M}$ PA. Expression levels of p-p65 were determined by western blot analysis. Cells were pretreated with MTX or Sal for 30 min prior to incubation with $100 \mu \mathrm{M}$ PA for $24 \mathrm{~h}$, and the release of (B) TNF- $\alpha$ and (C) IL-6 were determined by ELISA. (D) Adiponectin mRNA expression was determined by quantitative polymerase chain reaction. Sal served as a positive control. Data are expressed as the mean \pm standard deviation. ${ }^{~} \mathrm{P}<0.05$ vs. the blank group (no drug treatment); ${ }^{*} \mathrm{P}<0.05$ vs. the control group (PA treatment). MTX, methotrexate; PA, palmitic acid; Sal, salicylate; p, phosphorylated; TNF- $\alpha$, tumor necrosis factor- $\alpha$; IL-6, interleukin-6.

anti-inflammatory effect of MTX, 3T3-L1 cells were transfected with AMPKa1/2-specific siRNA to knockdown AMPK expression. As presented in Fig. 3A, silencing AMPK significantly decreased the inhibitory effect of MTX on PA-mediated $\mathrm{NF}-\kappa \mathrm{B}$ p65 phosphorylation $(\mathrm{P}<0.05)$. MTX treatment altered the production of TNF- $\alpha$ (Fig. 3B), IL-6 (Fig. 3C) and adiponectin (Fig. 3D) upon PA challenge, however, this action was also blocked by knockdown of AMPK (P<0.05, Fig. 3B-D). These results indicated that MTX inhibited inflammation in a AMPK-dependent manner.

Treatment of PVAT with MTX restores the loss of ACh-induced vasodilation. Endothelial dysfunction is characterized by the loss of endothelium-dependent vasodilation. To investigate the influence of PVAT on vessel function, PVAT was incubated with PA and collected the medium as CM to stimulate the rat aorta. Data presented in Fig. 4A indicated that ACh induced vasodilation, whereas stimulation with PA led to a significant loss in vessel relaxation $(\mathrm{P}<0.05)$. Pretreatment of PVAT with MTX prevented the alterations and effectively restored the loss of ACh-mediated vessel relaxation in a concentration-dependent manner, but this action was attenuated by co-treatment with AMPK inhibitor compound $\mathrm{C}(\mathrm{P}<0.05$, Fig. 4A). Furthermore, it was observed that treating PVAT with MTX effectively normalized eNOS phosphorylation in the aorta subjected to
CM challenge $(\mathrm{P}<0.05$, Fig. 4B). AMPK inhibitor compound $\mathrm{C}$ blocked the actions of MTX in the regulation of vasodilation and eNOS phosphorylation, suggesting the involvement of AMPK in the mechanism of MTX action. These results suggested that MTX improved vessel function via regulation of AMPK activity.

\section{Discussion}

Endothelial homeostasis is important in the regulation of vessel function and may predict the development of cardiovascular diseases independently of other known risk factors (13). PVAT is a functional component of the vasculature, exerting paracrine influences on endothelial homeostasis. A previous study has investigated the regulation of vessel tone by PVAT, attempting to identify adipocyte-derived relaxation and constriction factors, which are endothelium-independent (14). Inflammation in PVAT induces dysregulation of adipocytokine expression and subsequently impairs the integrity of the endothelium, which results in its dysfunction. The present study prepared an ex vivo model of PVAT/endothelial dysfunction by treating rat aorta with PVAT-derived CM and successfully observed the beneficial effects of MTX on adipocytokine expression and further implications in endothelial function. 
A
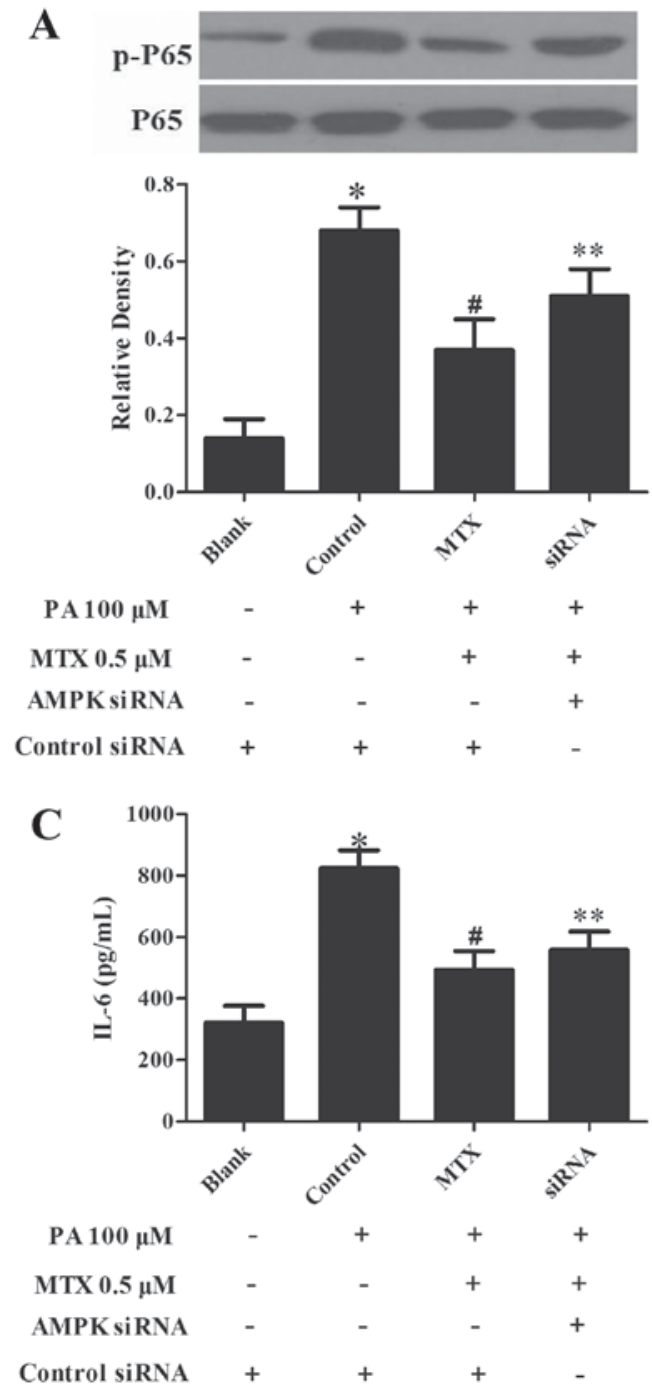

B

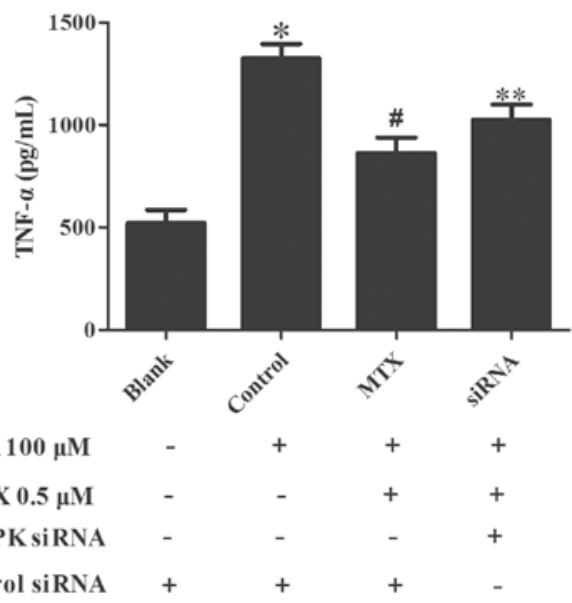

D

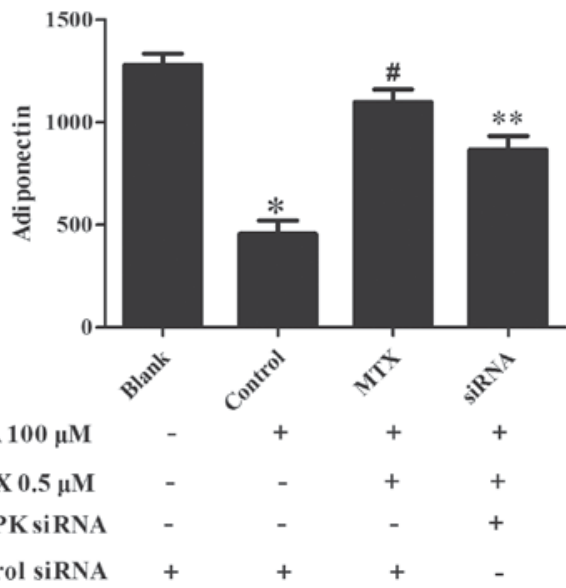

Figure 3. AMPK siRNA transfection impairs the ability of MTX to suppress inflammation in 3T3-L1 pre-adipocytes. (A) 3T3-L1 pre-adipocytes were transfected with AMPK $\alpha 1 / 2$ siRNA or control siRNA. siRNA-transfected adipocytes were pretreated with MTX for 30 min, then stimulated with $100 \mu \mathrm{M}$ PA for a further $30 \mathrm{~min}$. Nuclear factor- $\mathrm{\kappa}$ $\mathrm{p} 65$ phosphorylation was assayed by western blot analysis. Following transfection, cells were incubated with MTX in the presence of $100 \mu \mathrm{M}$ PA for $2 \mathrm{~h}$, then cultured in fresh medium for a further $22 \mathrm{~h}$, and the concentrations of (B) TNF- $\alpha$, (C) IL-6 and (D) adiponectin in the supernatant were measured with ELISA kits. Data are expressed as the mean \pm standard deviation. ${ }^{*} \mathrm{P}<0.05$ vs. Blank; ${ }^{*} \mathrm{P}<0.05$ vs. control; ${ }^{* *} \mathrm{P}<0.05$ vs. AMPK siRNA treatment. MTX, methotrexate; PA, palmitic acid; p, phosphorylated; TNF- $\alpha$, tumor necrosis factor- $\alpha$; IL-6, interleukin-6; AMPK, adenosine monophosphate-activated kinase; siRNA, small interfering RNA.

In addition to regulating energy metabolism, AMPK exerts anti-inflammatory activity, and this action has been implicated in the normalization of adipose and endothelial functions. The present study first investigated the role of MTX in the regulation of AMPK activation in PVAT, and observed that AMPK activity was increased by enhancing phosphorylation. PA stimulation was demonstrated to reduce AMPK phosphorylation in PVAT, consistent with results from a previous study (15).

It has previously been demonstrated that nanomolar concentrations of NO have anti-inflammatory and protective properties that are mediated by the inhibition of the activation of NF- $\mathrm{B}$ (16). PA stimulation evoked inflammation in PVAT, which induced adipose dysfunction as demonstrated by enhanced NF- $\kappa$ B p 65 phosphorylation. MTX attenuated NF- $\kappa B$ p 65 phosphorylation, suggesting the inhibition of $\mathrm{NF}-\kappa \mathrm{B}$ inflammatory signaling. This finding is consistent with a previous study in which PA induced NF- $\mathrm{KB}$-dependent inflammation by binding to toll-like receptor 4 in the endothelium (17). The expression levels of pro-inflammatory adipocytokines, including TNF- $\alpha$ and IL-6, were increased, whereas gene expression levels of adiponectin were downregulated. MTX reversed the alteration of adipocytokine expression, exhibiting a similar effect to that induced by salicylate, demonstrating its anti-inflammatory potency in the endothelium. To further elucidate the role of AMPK in the anti-inflammatory activity of MTX, the activity of MTX in adipocytes was observed. AMPK $\alpha$ knockdown using siRNA diminished the inhibitory effect of MTX on NF- $\mathrm{BB}$ activation and blocked its beneficial regulation of TNF- $\alpha$, IL- 6 and adiponectin production, further verifying the role of AMPK in the anti-inflammatory activity of MTX.

Endothelial dysfunction is characterized by the loss of endothelium-dependent vasodilation $(18,19)$. eNOS-derived $\mathrm{NO}$, which is a gaseous signaling molecule, is important in the maintenance of vascular homeostasis by promoting 


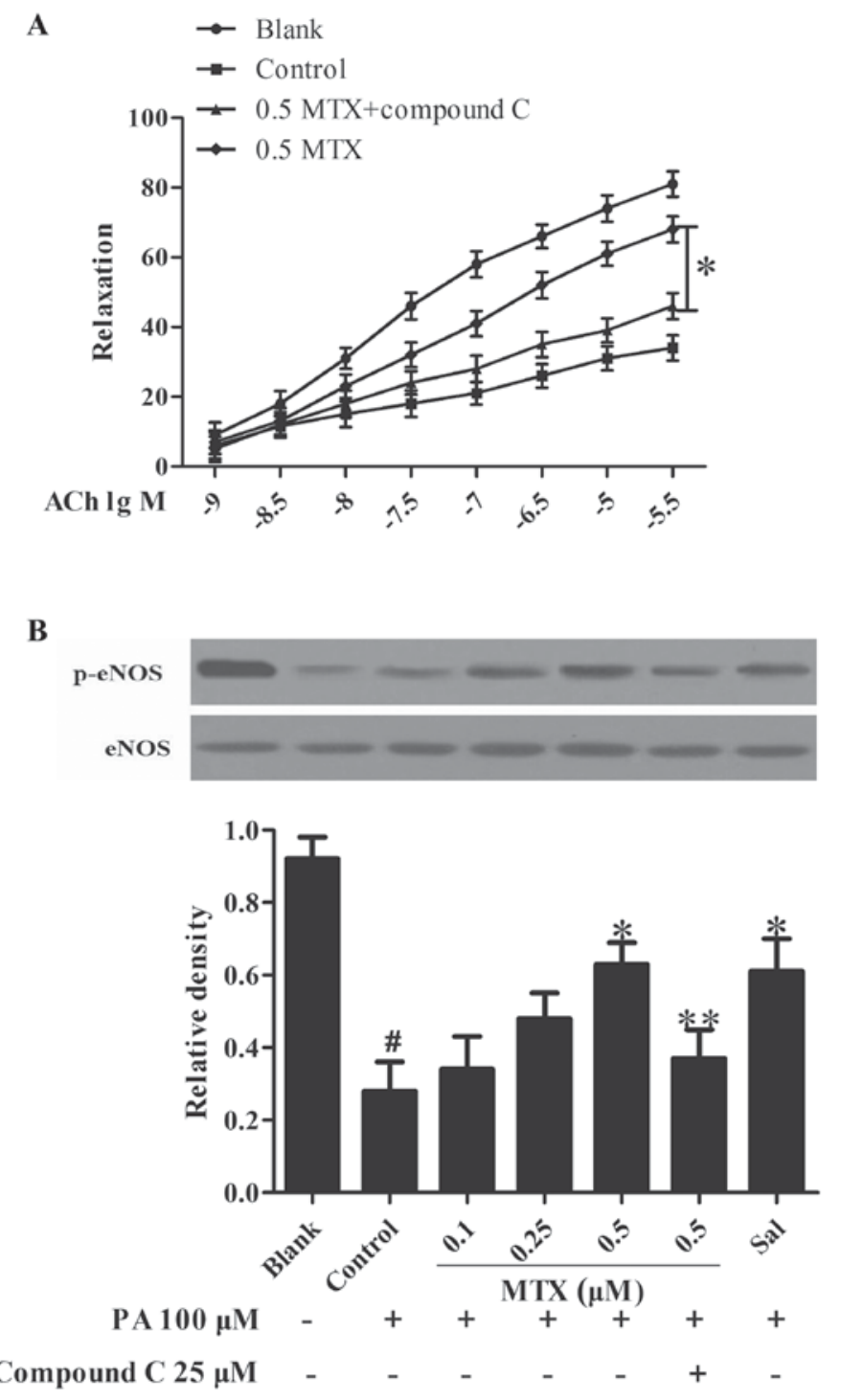

Figure 4. Effects of MTX on vasodilation and eNOS phosphorylation in CM-treated rat aorta. (A) CM was prepared by stimulating perivascular adipose tissue with $100 \mu \mathrm{M}$ PA in the presence or absence of MTX and compound C. Vasodilation was assessed by exposure to ACh. (B) The aorta was exposed to this CM for $30 \mathrm{~min}$ and eNOS phosphorylation was assayed by western blot analysis. Data are expressed as the mean \pm standard deviation of three independent experiments. ${ }^{~} \mathrm{P}<0.05$ vs. blank; ${ }^{*} \mathrm{P}<0.05$ vs. control; ${ }^{* *} \mathrm{P}<0.05$ vs. compound $\mathrm{C}$ treatment. MTX, methotrexate; $\mathrm{PA}$, palmitic acid; $\mathrm{p}$, phosphorylated; CM, conditioned medium; ACh, acetylcholine; eNOS, endothelial nitric oxide synthase.

vasodilation and inhibiting inflammation (20). Therefore, in order to observe the effects of AMPK agents on endothelial homeostasis via regulation of PVAT function, the present study stimulated PVAT with MTX and collected the medium as $\mathrm{CM}$ to treat rat aorta. Furthermore, ACh-induced endothelium-dependent vasodilation is mediated via eNOS activation and subsequent NO production. Pretreatment of PVAT with PA reduced eNOS phosphorylation and impaired ACh-mediated vasodilation, indicating the association between dysregulation of adipocy tokine expression and endothelial dysfunction. Co-treatment with the AMPK inhibitor compound $\mathrm{C}$ blocked the action of MTX on vasodilation, further demonstrating the involvement of AMPK in its regulation.

In conclusion, the present study established an ex vivo model of PVAT/endothelial dysfunction by stimulating rat aorta with CM derived from PVAT. It was demonstrated that pharmacological activators of AMPK regulated adipocytokine expression by inhibiting PVAT inflammation, and thereby ameliorated endothelial dysfunction in an AMPK interdependent manner. These findings provide a novel insight into the potential underlying mechanism by which MTX protects endothelial function against inflammatory insult.

\section{References}

1. World Health Statistics 2012. World Health Organization, Geneva, 2012.

2. Vita JA and Keaney JF: Endothelial function: A barometer for cardiovascular risk? Circulation 106: 640-642, 2002.

3. Li AC and Glass CK: The macrophage foam cell as a target for therapeutic intervention. Nat Med 8: 1235-1242, 2002.

4. Bijland S, Mancini SJ and Salt IP: Role of AMP-activated protein kinase in adipose tissue metabolism and inflammation. Clin Sci (Lond) 124: 491-507, 2013. 
5. Gauthier MS, O'Brien EL, Bigornia S, Mott M, Cacicedo JM Xu XJ, Gokce N, Apovian C and Ruderman N: Decreased AMP-activated protein kinase activity is associated with increased inflammation in visceral adipose tissue and with whole-body insulin resistance in morbidly obese humans. Biochem Bioph Res Commun 404: 382-387, 2011.

6. Tsai KL, Chen LH, Chiou SH, Chiou GY, Chen YC, Chou HY, Chen LK, Chen HY, Chiu TH, Tsai CS, et al: Coenzyme Q10 suppresses oxLDL-induced endothelial oxidative injuries by the modulation of LOX-1-mediated ROS generation via the AMPK/PKC/NADPH oxidase signaling pathway. Mol Nutr Food Res 55 (Suppl 2): S227-S240, 2011.

7. Sun Y, Li J, Xiao N, Wang M, Kou J, Qi L, Huang F, Liu B and Liu K: Pharmacological activation of AMPK ameliorates perivascular adipose/endothelial dysfunction in a manner interdependent on AMPK and SIRT1. Pharmacol Res 89: 19-28, 2014.

8. Saag KG, Teng GG, Patkar NM, Anuntiyo J, Finney C, Curtis JR, Paulus HE, Mudano A, Pisu M, Elkins-Melton M, et al: American College of Rheumatology 2008 recommendations for the use of nonbiologic and biologic disease-modifying antirheumatic drugs in rheumatoid arthritis. Arthritis Rheum 59: 762-784, 2008.

9. Ridker PM: Testing the inflammatory hypothesis of atherothrombosis: Scientific rationale for the cardiovascular inflammation reduction trial (CIRT). J Thromb Haemost 7 (Suppl 1): S332-S339, 2009.

10. Bulgarelli A, Martins Dias AA, Caramelli B and Maranhão RC: Treatment with methotrexate inhibits atherogenesis in cholesterol-fed rabbits. J Cardiovasc Pharmacol 59: 308-314, 2012.

11. Livak KJ and Schmittgen TD: Analysis of relative gene expression data using real-time quantitative PCR and the 2(-Delta Delta C(T)) method. Methods 25: 402-408, 2001.
12. Mugabo Y, Mukaneza Y and Renier G: Palmitate induces C-reactive protein expression in human aortic endothelial cells. Relevance to fatty acid-induced endothelial dysfunction. Metabolism 60: 640-648, 2011.

13. Calles-Escandon J and Cipolla M: Diabetes and endothelial dysfunction: A clinical perspective. Endocr Rev 22: 36-52, 2001.

14. Gollasch M: Vasodilator signals from perivascular adipose tissue. Br J Pharmacol 165: 633-642, 2012.

15. Steinberg GR, Michell BJ, van Denderen BJ, Watt MJ, Carey AL, Fam BC, Andrikopoulos S, Proietto J, Görgün CZ, Carling D, et al: Tumor necrosis factor alpha-induced skeletal muscle insulin resistance involves suppression of AMP-kinase signaling. Cell Metab 4: 465-474, 2006.

16. Peng HB, Libby P and Liao JK: Induction and stabilization of I kappa B alpha by nitric oxide mediates inhibition of NF-kappa B. J Biol Chem 270: 14214-14219, 1995.

17. Maloney E, Sweet IR, Hockenbery DM, Pham M, Rizzo NO, Tateya S, Handa P, Schwartz MW and Kim F: Activation of NF-kappaB by palmitate in endothelial cells: A key role for NADPH oxidase-derived superoxide in response to TLR4 activation. Arterioscl Throm Vas Biol 29: 1370-1375, 2009.

18. Félétou M and Vanhoutte PM: Endothelial dysfunction: A multifaceted disorder (The Wiggers Award Lecture). Am J Physiol Heart Circ Physiol 291: H985-H1002, 2006.

19. Cersosimo E and DeFronzo RA: Insulin resistance and endothelial dysfunction: The road map to cardiovascular diseases. Diabetes Metab Res Rev 22: 423-436, 2006.

20. Moncada S: Nitric oxide in the vasculature: Physiology and pathophysiology. Ann NY Acad Sci 811: 60-69, 1997. 